\title{
Measurement of metabolic tumor volume: static versus dynamic FDG scans
}

\author{
Patsuree Cheebsumon ${ }^{1}$, Floris HP van Velden ${ }^{1}$, Maqsood Yaqub ${ }^{1}$, Corneline J Hoekstra² ${ }^{2}$ Linda M Velasquez ${ }^{3}$, \\ Wendy Hayes ${ }^{3}$, Otto S Hoekstra ${ }^{1}$, Adriaan A Lammertsma ${ }^{1}$ and Ronald Boellaard ${ }^{1 *}$
}

\begin{abstract}
Background: Metabolic tumor volume assessment using positron-emission tomography [PET] may be of interest for both target volume definition in radiotherapy and monitoring response to therapy. It has been reported, however, that metabolic volumes derived from images of metabolic rate of glucose (generated using Patlak analysis) are smaller than those derived from standardized uptake value [SUV] images. The purpose of this study was to systematically compare metabolic tumor volume assessments derived from SUV and Patlak images using a variety of (semi-)automatic tumor delineation methods in order to identify methods that can be used reliably on (whole body) SUV images.

Methods: Dynamic $\left[{ }^{18} \mathrm{~F}\right]$-fluoro-2-deoxy-D-glucose [FDG] PET data from 10 lung and 8 gastrointestinal cancer patients were analyzed retrospectively. Metabolic tumor volumes were derived from both Patlak and SUV images using five different types of tumor delineation methods, based on various thresholds or on a gradient.

Results: In general, most tumor delineation methods provided more outliers when metabolic volumes were derived from SUV images rather than Patlak images. Only gradient-based methods showed more outliers for Patlakbased tumor delineation. Median measured metabolic volumes derived from SUV images were larger than those derived from Patlak images (up to 59\% difference) when using a fixed percentage threshold method. Tumor volumes agreed reasonably well ( $<26 \%$ difference) when applying methods that take local signal-to-background ratio [SBR] into account.

Conclusion: Large differences may exist in metabolic volumes derived from static and dynamic FDG image data. These differences depend strongly on the delineation method used. Delineation methods that correct for local SBR provide the most consistent results between SUV and Patlak images.
\end{abstract}

Keywords: tumor delineation, tumor volume, FDG PET, Patlak, SUV

\section{Background}

Positron-emission tomography [PET] may be used to delineate the biological target volume for both radiotherapy and response monitoring purposes [1-4]. The most widely used PET tracer, $\left[{ }^{18} \mathrm{~F}\right]$-fluoro-2-deoxy-Dglucose [FDG], might improve accuracy of tumor volume definition for radiotherapy by identifying areas within the tumor that are more metabolically active [5]. Tumor volumes can be delineated on either images of glucose metabolic rate or standardized uptake value

\footnotetext{
* Correspondence: r.boellaard@vumc.nl

'Department of Nuclear Medicine \& PET Research, VU University Medical

Center, P.O. Box 7057, Amsterdam, 1007 MB, The Netherlands

Full list of author information is available at the end of the article
}

[SUV] images [6]. SUV is most commonly used for (semi-)quantification of whole-body FDG PET studies and only requires a static scan. Images of glucose metabolic rate can be generated from dynamic scans using a measured or image-derived arterial input function together with Patlak graphical analysis. It is well known that Patlak analysis is quantitatively more accurate than SUV analysis. Patlak analysis, however, requires a dynamic scan and limits data acquisition to a single bed position with an axial coverage of $<20 \mathrm{~cm}$.

As shown previously [6], metabolic volumes, defined using a $50 \%$ isocontour method, were smaller when defined on Patlak images than when defined on SUV images. To date, however, no systematic comparison has 
been performed in which various existing (semi-)automatic tumor delineation methods have been applied to both SUV and Patlak images.

Recently, a number of different (semi-)automatic tumor delineation methods have been validated for SUV images using both simulations $[7,8]$ and lung tumor FDG scans $[9,10]$. Most methods showed good performance as measured maximum diameters derived from these methods corresponded well with pathological measurements [11]. As most of tumor delineation methods that correct for local background are less sensitive to changes in local contrast, these methods might show better correspondence between measured tumor volumes derived from either Patlak or SUV images. Therefore, the purpose of this study was to systematically compare measured metabolic tumor volumes derived from SUV and Patlak images using a variety of (semi-)automatic tumor delineation methods.

\section{Materials and methods Patient data}

Dynamic FDG PET scans from 10 non-small cell lung cancer [NSCLC] (stages IIIB to IV) patients [12] and 8 gastrointestinal [GI] (colorectal carcinoma) cancer patients [13] were included retrospectively. All scans had been acquired prior to therapy. All patients had given written informed consent, and both studies had been approved by the Medical Ethics Review Committee of the VU University Medical Center.

For NSCLC patients (three females, seven males; weight $76 \pm 10 \mathrm{~kg}$, range 56 to $94 \mathrm{~kg}$ ), blood glucose levels were within the normal range (mean $5.5 \pm 0.6 \mathrm{mmol} \cdot \mathrm{L}^{-1}$, range 4.4 to $\left.7.0 \mathrm{mmol} \cdot \mathrm{L}^{-1}\right)$. The same was true for blood glucose levels (mean $5.6 \pm 0.8 \mathrm{mmol} \cdot \mathrm{L}^{-1}$, range 3.9 to $7.0 \mathrm{mmol} \cdot \mathrm{L}^{-1}$ ) of patients with advanced GI malignancies (one female, seven males; weight $85 \pm 15 \mathrm{~kg}$, range 60 to $110 \mathrm{~kg}$ ).

\section{PET scanning protocol}

All patients fasted for at least $6 \mathrm{~h}$ before scanning. Patients were prepared in accordance with recently published guidelines for quantitative PET studies [14]. They were scanned in a supine position and received an intravenous catheter for tracer administration. During dynamic scanning, blood samples for determining plasma glucose levels were collected at fixed times (i.e., at 35, 45, 55 min post injection). All dynamic scans were performed using an ECAT EXACT HR+ scanner (Siemens/ CTI, Knoxville, TN, USA) [15], having a $15.5-\mathrm{cm}$ axial field of view. Each scan session started with a 10-min transmission scan using three retractable rotating ${ }^{68} \mathrm{Ge}$ line sources. After completion of the transmission scan, a bolus of FDG was administrated intravenously (388 \pm 71 and $459 \pm 97 \mathrm{MBq}$ for NSCLC and GI cancer, respectively), at the same time starting a dynamic emission scan in a 2-D acquisition mode. Each dynamic scan consisted of 40 frames with the following lengths, $1 \times 30,6 \times 5,6 \times$ $10,3 \times 20,5 \times 30,5 \times 60,8 \times 150,6 \times 300$ s. In addition, a static scan was created by summing the sinograms of the last three frames (i.e., 45 to $60 \mathrm{~min}$ post injection).

All data were normalized and corrected for attenuation, random coincidences, scatter radiation, dead time, and decay. Reconstructions were performed using normalization and attenuation-weighted ordered subsets expectation maximization [OSEM] with 2 iterations and 16 subsets, followed by post-smoothing using a 0.5 Hanning filter. This resulted in an image resolution of approximately $6.5 \mathrm{~mm}$ full width at half maximum. An image matrix size of $256 \times 256 \times 63$ was used, corresponding to a pixel size of $2.57 \times 2.57 \times 2.43 \mathrm{~mm}^{3}$.

After reconstruction, the summed image ( 45 to 60 min post injection) was used to generate a SUV image by normalizing local tissue concentrations to injected dose and body weight. In addition, Patlak analysis, a kinetic linearized model [16] for irreversible tracer uptake, was applied to the interval 10 to $60 \mathrm{~min}$ post injection to generate an image of net influx rate $\left[K_{\mathrm{i}}\right]$ of FDG, which is proportional to the metabolic rate of glucose. Image-derived input functions [IDIF] were used as plasma input curves and obtained as described by Cheebsumon et al. [17]. In short, 3-D volumes of interest [VOI] were drawn manually in three vascular structures (i.e., the left ventricle, aortic arch, and ascending aorta) using an early frame that highlights the blood pool [18]. These VOI were then projected onto all frames yielding arterial whole blood time activity curves (i.e., IDIF). The average input curves from VOI defined in the three vascular structures were used as an input function during Patlak analysis.

\section{Data analysis}

For the 10 NSCLC patients, VOI were defined for 54 lesions that were all located in the lung. For the $8 \mathrm{GI}$ cancer patients, VOI were defined for 37 lesions that were located in the liver $(n=23)$, lung $(n=12)$, or colon $(n=2)$. All lesions that could be identified by an expert physician were included in this study. Metabolic tumor volumes were obtained using the following five different types of (semi-)automatic VOI methods:

Fixed threshold of $50 \%$ and $70 \%\left(\mathrm{VOI}^{50}, \mathrm{VOI}^{70}\right)$. In this method, a fixed threshold (i.e., $50 \%$ or $70 \%$ ) of the maximum voxel value within a tumor is used to delineate the tumor [19].

Adaptive threshold of $41 \%, 50 \%$, and $70 \%\left(\mathrm{VOI}^{A 41}\right.$, $\left.V O I^{A 50}, V O I^{A 70}\right)$. This is similar to the fixed threshold method, except that it adapts the threshold relative to the local average background, thereby correcting for the contrast between the tumor and local background [19]. 
Contrast-oriented method (VOI $\left.{ }^{\text {Schaefer }}\right)$. This method uses the average of SUV within a 70\% threshold of $\mathrm{SUV}_{\max }$ isocontour (meanSUV $\mathrm{V}_{70 \%}$ ) and background activity for various sphere sizes. Regression coefficients are calculated, which represent the relationship between the optimal threshold and image contrast for various sphere sizes [3]. This threshold equation is given by:

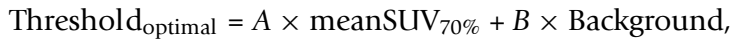

where $A$ and $B$ are fitted using phantom studies [3]. When applied to Patlak images, $K_{\mathrm{i}}$ rather than SUV is used. In general, different values are applied for sphere diameters smaller and larger than $3 \mathrm{~cm}$ in diameter. In the present study, this method was recalibrated, i.e., specific $A$ and $B$ values for the image characteristics used were determined.

Background-subtracted relative-threshold level [RTL] method $\left(V O I^{R T L}\right)$. This method is an iterative method that is based on a convolution of the point-spread function, which takes into account differences between various sphere sizes and the scanner resolution [4].

Gradient-based watershed segmentation method. This method uses two steps before calculating the VOI [2]. First, a gradient image is calculated on which a 'seed' is placed in the tumor (tumor basin) and another in the background (background basin). Next, a watershed [WT] algorithm is used to grow both seeds in the gradient basins, thereby creating boundaries on the gradient edges. In the present study, two different types of gradient basins were used. In the first approach [ $\left.\mathrm{Grad}^{\mathrm{WT1}}\right]$, all voxels on the edge between the tumor and background are assigned to the tumor $[8,10]$. In the second approach [Grad ${ }^{\text {WT2 }}$ ], an upsampled image is used to ensure less effects of sampling. In addition, a voxel on the edge between the tumor and background is allocated to either the tumor or background based on the smallest difference with that voxel value.

To reduce sensitivity to noise, for all methods, the maximum voxel value was obtained using a crossshaped pattern. This method searches for the region with the (local) average maximum intensity based on the average of seven neighboring voxels, which was then used as a maximum or 'peak' value. The tumor-to-background ratio was calculated by dividing this maximum value by the background value surrounding the tumor.

\section{Statistical analyses}

Both metabolic volumes and differences in measured volumes derived from two image types are reported. The percentage volume difference was defined as $\left(\frac{\text { Volume }_{\text {Suv }}}{\text { Volume }_{\text {Patlak }}}-1\right) \times 100 \%$. Note that this value can be negative, indicating an underestimation of the SUV- derived metabolic volume compared with the Patlakderived volume. In addition, for each delineation method, mean, median, first quartile, third quartile, minimum, and maximum values, including statistical outliers, are reported in box plots. Moreover, visual outliers were identified as VOIs that showed unrealistically large or small volumes when compared visually with the tumor. These outliers were not included in the statistical analysis when calculating $p$ values. A two-tailed Wilcoxon signed-rank test was used to indicate statistically significant differences between measured volumes derived from SUV and Patlak images, where $p$ values less than 0.05 were considered to be significant.

\section{Results}

Table 1 shows the number of visual outliers (i.e., those cases where there is an obvious mismatch between derived VOI and tumor boundaries) for all methods applied to both Patlak and SUV images, specifying results for NSCLC and GI cancer separately. In general, most tumor delineation methods provided more outliers when metabolic volumes were derived from SUV images rather than Patlak images. Only gradient-based methods showed more outliers for Patlak-based tumor delineation. $\mathrm{VOI}^{70}$ and $\mathrm{VOI}^{\mathrm{A} 70}$ provided no outliers for either image or cancer type.

In general, measured tumor volumes derived from SUV images were larger than those derived from Patlak images. Example images of the measured tumor volumes derived from SUV and Patlak images are shown in Figure 1. Exceptions were $\mathrm{VOI}^{\mathrm{A} 70}$ for both types of cancer and the two gradient-based methods for GI cancer (Tables 2 and 3). Large differences (up to $58.7 \%$ and $28.1 \%$ for NSCLC and GI cancer, respectively) in measured metabolic volume based on the two image types were observed for

Table 1 Number of visual outliers in SUV- and Patlakderived measured metabolic volumes for both cancer

\begin{tabular}{lcccc}
\hline $\begin{array}{l}\text { Delineation } \\
\text { method }\end{array}$ & \multicolumn{2}{c}{ NSCLC } & \multicolumn{2}{c}{ Gl cancer } \\
& $\begin{array}{c}\text { SUV } \\
\text { image }\end{array}$ & $\begin{array}{c}\text { Patlak } \\
\text { image }\end{array}$ & $\begin{array}{c}\text { SUV } \\
\text { image }\end{array}$ & $\begin{array}{c}\text { Patlak } \\
\text { image }\end{array}$ \\
\hline VOI $^{50}$ & 9 & - & 5 & 3 \\
VOI $^{70}$ & - & - & - & - \\
VII $^{\text {A41 }}$ & 4 & 1 & 5 & 3 \\
VOI $^{\text {A50 }}$ & - & - & - & 1 \\
VOI $^{\text {A70 }}$ & - & - & - & - \\
VOI $^{\text {RTL }}$ & - & 1 & 3 & - \\
VOI $^{\text {Schaefer }}$ & 2 & 2 & 3 & 2 \\
Grad $^{\text {WT1 }}$ & - & 3 & 13 & 15 \\
Grad $^{\text {WT2 }}$ & 2 & 5 & 5 & 5 \\
\hline
\end{tabular}

NSCLC, non-small cell lung cancer; GI, gatrointestinal; SUV, standardized uptake value; VOI, volumes of interest; Grad ${ }^{\mathrm{WT} 1}$, gradient-based watershed first approach; Grad ${ }^{\text {WT2 }}$, gradient-based watershed second approach. 


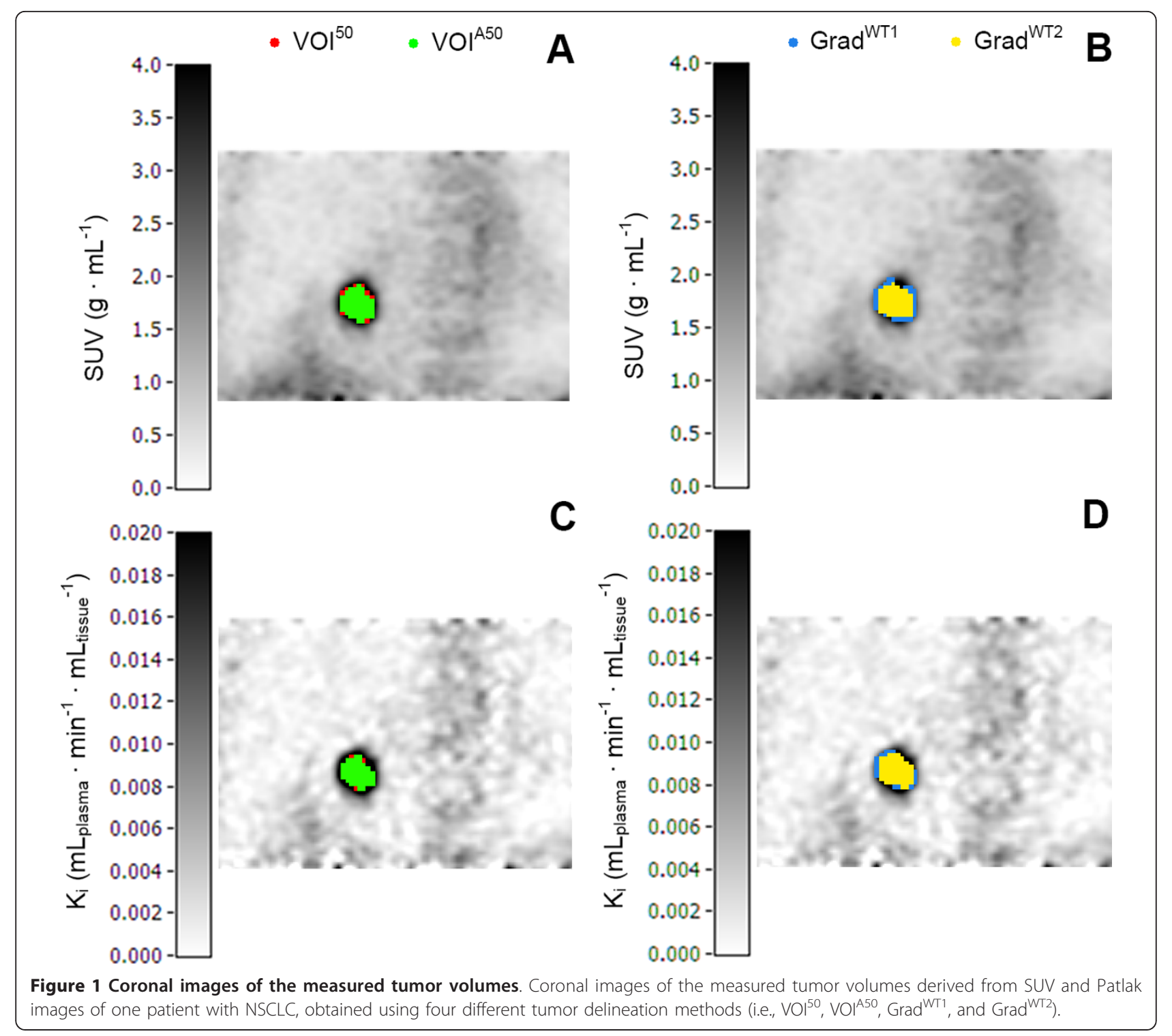

Table 2 Mean, median, minimum, and maximum values of metabolic volumes and their median differences for NSCLC Delineation method Volume obtained from SUV image Volume obtained from Patlak image $\%$ Median difference ${ }^{\mathrm{a}} P$ value $P$ value ${ }^{\mathrm{b}}$ $(\mathrm{mL}) \quad(\mathrm{mL})$

\begin{tabular}{|c|c|c|c|c|c|c|c|c|c|c|c|}
\hline & Mean & Median & Min & Max & Mean & Median & Min & Max & & & \\
\hline$\overline{\left.\mathrm{VO}\right|^{50}}$ & 84.2 & 6.9 & 1.2 & 950.2 & 7.7 & 2.7 & 0.7 & 74.4 & 58.7 & $<0.001$ & $<0.001$ \\
\hline $\mathrm{VOl}^{70}$ & 4.9 & 1.5 & 0.5 & 50.2 & 2.5 & 1.1 & 0.3 & 23.7 & 48.0 & $<0.001$ & $<0.001$ \\
\hline $\mathrm{VOI} \mathrm{A}^{\mathrm{A} 41}$ & 22.7 & 5.2 & 1.2 & 294.4 & 10.9 & 3.5 & 0.9 & 95.2 & 25.8 & $<0.001$ & $<0.001$ \\
\hline $\mathrm{VOI}^{\mathrm{A} 50}$ & 8.8 & 2.4 & 0.6 & 97.0 & 6.6 & 2.4 & 0.5 & 70.6 & 15.0 & $<0.001$ & $<0.001$ \\
\hline $\mathrm{VOI} \mathrm{A}^{\mathrm{A} 70}$ & 2.1 & 0.5 & 0.1 & 23.4 & 1.8 & 0.8 & 0.2 & 19.9 & -25.0 & 0.044 & 0.044 \\
\hline$V O I^{R T L}$ & 10.4 & 3.6 & 0.4 & 101.9 & 7.7 & 3.1 & 0.3 & 73.1 & 15.6 & $<0.001$ & $<0.001$ \\
\hline $\mathrm{VOI}^{\text {schaefer }}$ & 17.2 & 5.1 & 1.0 & 125.7 & 13.8 & 4.2 & 0.9 & 104.2 & 14.3 & $<0.001$ & $<0.001$ \\
\hline Grad $^{\text {WT1 }}$ & 12.3 & 3.9 & 1.4 & 163.2 & 10.4 & 5.4 & 1.6 & 50.9 & 1.9 & 0.725 & 0.324 \\
\hline Grad $^{\mathrm{WT2}}$ & 5.8 & 2.7 & 0.6 & 63.2 & 3.7 & 2.1 & 0.5 & 28.2 & 18.2 & $<0.001$ & 0.001 \\
\hline
\end{tabular}

${ }^{\mathrm{a}}$ The percentage difference was defined as $\left(\frac{\text { Volume }_{\text {SUV }}}{\text { Volume }_{\text {Patlak }}}-1\right) \times 100 \%$. The average tumor-to-background ratio was 5.3 (range 2.7 to 12.7$)$ and 18.8 (range 6.1 to 81.0 ) when derived from SUV and Patlak images, respectively. ${ }^{b}$ Without visual outliers. SUV, standardized uptake value; min, minimum; max, maximum; VOI, volumes of interest; Grad ${ }^{\text {WT1 }}$, gradient-based watershed first approach; Grad ${ }^{\text {WT2 }}$, gradient-based watershed second approach. 
Table 3 Mean, median, minimum, and maximum values of metabolic volumes and median differences for GI cancer

\begin{tabular}{|c|c|c|c|c|c|c|c|c|c|c|c|}
\hline \multirow[t]{2}{*}{ Delineation method } & \multicolumn{4}{|c|}{$\begin{array}{l}\text { Volume obtained from SUV image } \\
\qquad(\mathrm{mL})\end{array}$} & \multicolumn{4}{|c|}{$\begin{array}{l}\text { Volume obtained from Patlak image } \\
\qquad(\mathrm{mL})\end{array}$} & \multirow[t]{2}{*}{$\%$ Median difference $^{a}$} & \multirow[t]{2}{*}{$P$ value } & \multirow[t]{2}{*}{$P$ value $^{\mathrm{b}}$} \\
\hline & Mean & Median & Min & Max & Mean & Median & Min & Max & & & \\
\hline $\mathrm{VOI}^{50}$ & 190.4 & 15.4 & 2.7 & 2297.8 & 65.2 & 10.1 & 2.1 & 822.9 & 28.1 & $<0.001$ & $<0.001$ \\
\hline $\mathrm{VOI}^{70}$ & 10.5 & 3.9 & 1.3 & 57.6 & 8.8 & 3.5 & 1.0 & 45.2 & 18.5 & $<0.001$ & $<0.001$ \\
\hline $\mathrm{VOI}^{\mathrm{A} 41}$ & 195.4 & 28.6 & 3.3 & 2402.5 & 86.1 & 11.9 & 2.5 & 1257.6 & 16.5 & $<0.001$ & $<0.001$ \\
\hline $\mathrm{VOI} \mathrm{A}^{\mathrm{A} 0}$ & 20.3 & 6.0 & 2.1 & 121.2 & 22.2 & 6.8 & 1.9 & 107.3 & 8.1 & 0.364 & 0.215 \\
\hline $\mathrm{VOI} \mathrm{I}^{\mathrm{A} 70}$ & 5.1 & 1.7 & 0.7 & 38.8 & 6.57 & 2.38 & 0.51 & 34.26 & -13.3 & 0.001 & 0.001 \\
\hline $\mathrm{VOI} \mathrm{I}^{\mathrm{RTL}}$ & 33.3 & 7.2 & 0.3 & 538.2 & 17.78 & 6.75 & 0.26 & 111.14 & 7.5 & 0.040 & 0.042 \\
\hline $\mathrm{VOI}^{\text {Schaefer }}$ & 158.0 & 14.7 & 3.4 & 2212.0 & 48.1 & 13.0 & 2.5 & 564.6 & 8.3 & 0.003 & 0.004 \\
\hline Grad $^{\text {WT1 }}$ & 43.5 & 32.8 & 9.1 & 223.5 & 51.2 & 43.2 & 10.2 & 229.4 & -9.1 & 0.025 & 0.085 \\
\hline Grad $^{\text {WT2 }}$ & 12.4 & 6.8 & 1.5 & 74.1 & 14.2 & 8.6 & 1.6 & 85.3 & -2.1 & 0.625 & 1.000 \\
\hline
\end{tabular}

${ }^{a}$ The percentage difference was defined as $\left(\frac{\text { Volume }_{\text {SUV }}}{\text { Volume }_{\text {Patlak }}}-1\right) \times 100 \%$. The average tumor-to-background ratio was 7.4 (range 2.4 to 31.6 ) and 16.0

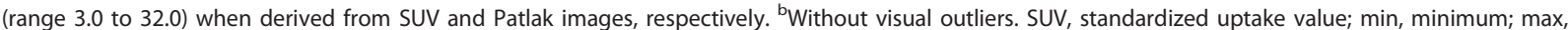
maximum; VOI, volumes of interest; Grad ${ }^{\text {WT1 }}$, gradient-based watershed first approach; Grad ${ }^{\text {WT2 }}$, gradient-based watershed second approach.

the various delineation methods (Figure 2A). In the case of NSCLC, the median difference in volume was higher for fixed threshold methods than for adaptive, contrastoriented, or gradient-based methods. This is further illustrated by Figure 3A, where $\mathrm{VOI}^{\mathrm{A} 50}$ (i.e., with background correction) shows better correspondence between SUVand Patlak-based volumes than $\mathrm{VOI}^{50}$ (i.e., without background correction). Only $\mathrm{Grad}^{\mathrm{WT} 1}$ provided no significant difference $(p>0.05)$ in the metabolic volume derived from SUV and Patlak images, but this may be due to the large spread in differences (Figure 3B). Similar results were found when these differences in volume were compared to SUV (or $K_{\mathrm{i}}$ values, Figures 4A, B). In general, we observed that smaller lesions also had the lowest SUV. Consequently, the largest volume differences between SUV and Patlak image-based tumor delineations were seen for lesions having a low SUV and a small metabolic volume.

Similar trends were observed for GI cancer (Figures 2B and $3 \mathrm{C}, \mathrm{D}$ ). Here, Grad ${ }^{\mathrm{WT} 1}$ (only after removal of visual outliers), $\mathrm{Grad}^{\mathrm{WT} 2}$, and $\mathrm{VOI}^{\mathrm{A} 50}$ provided no significant differences $(p>0.05)$ between measured volumes derived from both image types. In addition, similar trends were observed when data from both studies were pooled and presented separately for the specific locations of the tumors (i.e., the liver or the lung, Figure 2C).

\section{Discussion}

The main use of FDG is measurement of glucose metabolism. However, FDG PET can also be used to measure the volume with increased metabolism. In a previous report [6], it was shown that tumor delineation using Patlak-derived glucose metabolism images provided smaller volumes and sharper borders than when SUV images were used. This was due to a higher local contrast in Patlak images than in SUV images. Patlak analysis, however, may not always be feasible or optimal because it requires (measured) arterial input data and a dynamic scan, which limits coverage to a single bed position. Therefore, in clinical practice, a static wholebody scan (covering the whole body) might be preferred, in which case data can only be analyzed using a SUV approach. It is well known, however, that SUV may be affected by technical, biological, and physical factors [20] that could hamper tumor delineation using this image type.

In agreement with Visser et al. [6], the present study showed (for two types of cancer) that when a fixed percentage threshold method (i.e., $\mathrm{VOI}^{50}$ ) was used, significantly larger metabolic volumes were obtained from SUV images than from Patlak images. However, these differences reduced when using methods that correct for local background and/or contrast, and in the case of gradient-based methods (Figure 2, Tables 2 and 3). This confirms that SUV-based tumor delineation is sensitive to signal-tobackground ratios. Differences in Patlak- and SUV-derived volumes were larger for NSCLC than for GI cancer, especially in the case of methods that use a fixed percentage threshold without background correction. As local (image) contrast for GI cancer was larger than that for NSCLC (average tumor-to-background ratios 7.4 and 5.3, respectively), this further illustrates the sensitivity to signal-tobackground ratio.

Some tumor delineation methods (i.e., $\mathrm{VOI}^{50}, \mathrm{VOI}^{\mathrm{A} 41}$, and $\mathrm{Grad}^{\mathrm{WT} 1}$ ) provided visually, unrealistically large tumor volumes (in up to $41 \%$ of cases) when applied to SUV images, while $\mathrm{VOI}^{\text {Schaefer }}$ did the same (in up to $8 \%$ of cases) for both image types (Table 1). In contrast, Grad $^{\text {WT2 }}$ provided many unrealistically small tumor volumes (in up to $24 \%$ of cases) for both image types. This suggests that these methods should be applied 


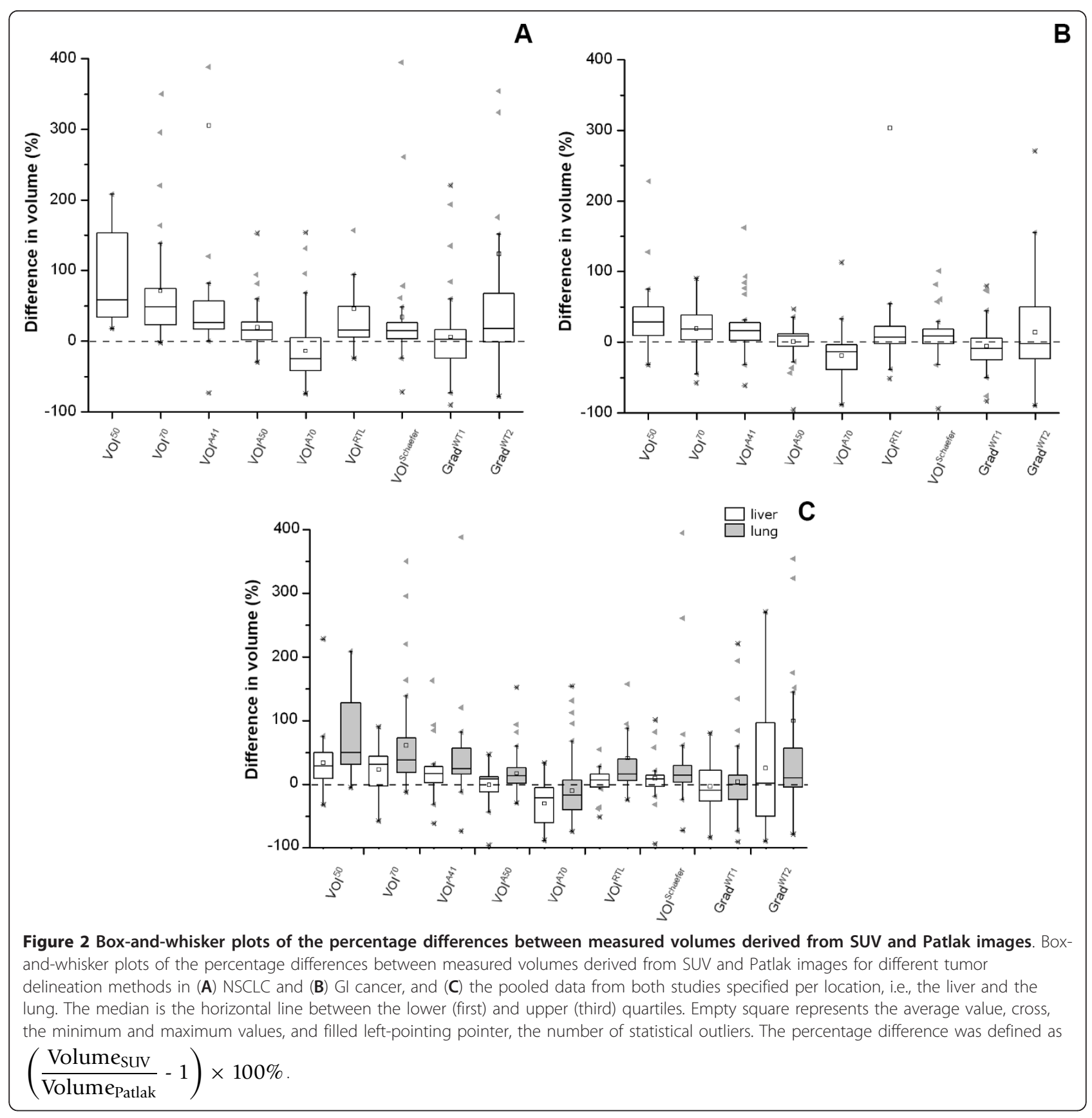

carefully and that their performance should be supervised.

Two different implementations of gradient-based methods were evaluated in the present study. In a previous NSCLC study [11], tumor diameters obtained using Grad $^{\text {WT2 }}$ corresponded better to pathology than those obtained using Grad ${ }^{\mathrm{WT} 1}$. As shown in Figure 3B, the present study also showed that measured volumes obtained from Grad $^{\text {WT2 }}$ were smaller than those from Grad $^{\text {WT1 }}$. However, Grad ${ }^{\text {WT1 }}$ showed better correspondence between SUV- and Patlak-derived volumes than
$\operatorname{Grad}^{\mathrm{WT} 2}(1.9 \%$ and $18.2 \%$, respectively). In contrast, for GI cancer, Grad ${ }^{\mathrm{WT} 2}$ showed better correspondence between SUV- and Patlak-derived volumes than $\operatorname{Grad}^{\text {WT1 }}(-2.1 \%$ and $-9.1 \%$, respectively). This suggests that the performance of gradient-based methods may also depend on signal-to-background ratios.

Differences between metabolic volumes obtained from SUV and Patlak images reduced when signal-to-background-corrected delineation methods are used. This finding is in line with previous studies reporting on testretest variability using various tumor delineation 


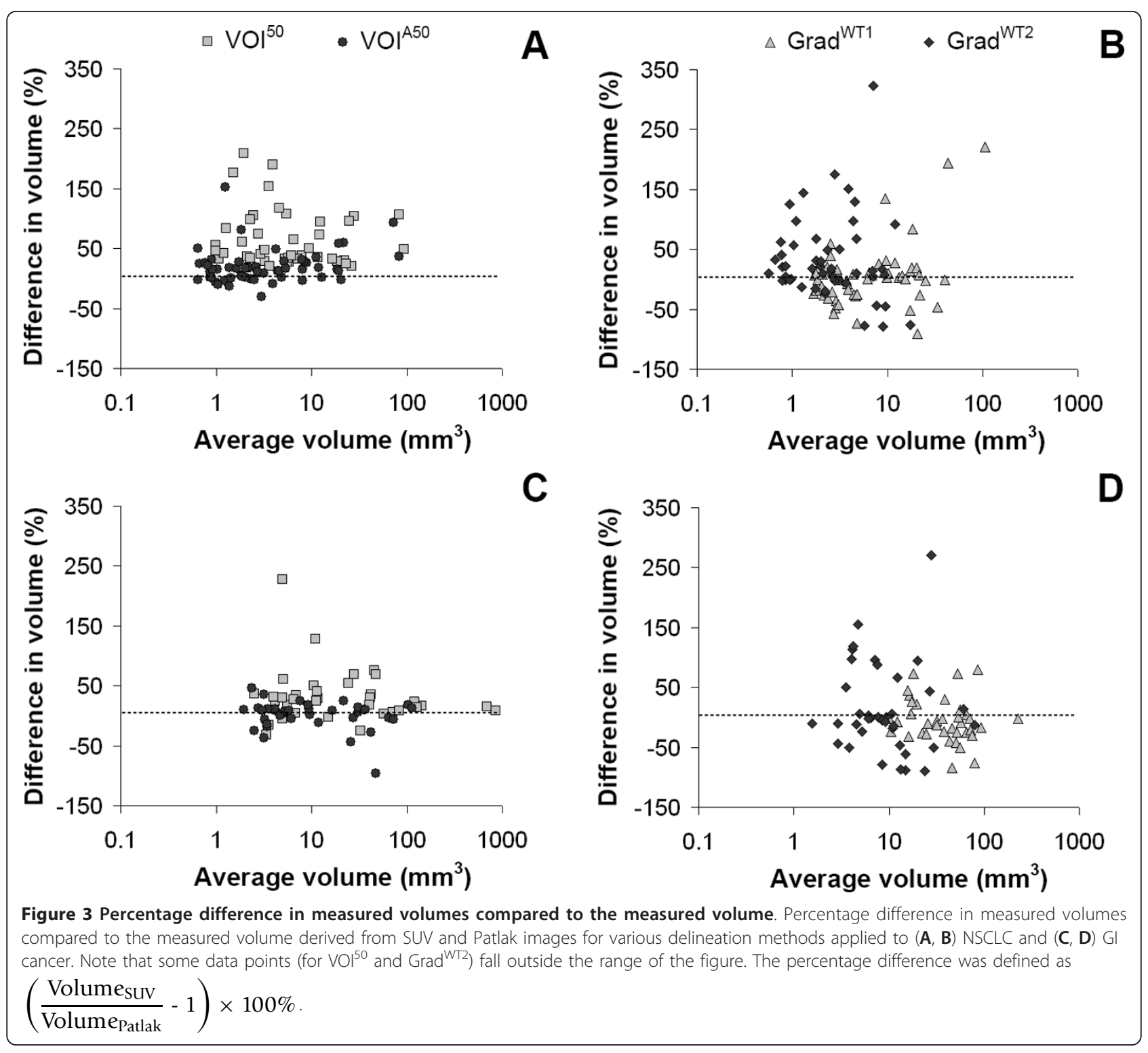

methods $[10,21]$ that confirmed that $\mathrm{VOI}^{\mathrm{A} 50}$ seems to be a good possible candidate for response monitoring purposes. However, gradient-based methods have been shown to be good candidates for radiotherapy purposes $[10,22]$. Therefore, either signal-to-background-corrected or gradient-based methods may be good candidates for response assessments and radiotherapy purposes.

\section{Limitations}

A limitation of this study is the lack of an independent reference standard to define tumor volumes, and consequently, in this paper, we could only study differences in (semi-)automatic tumor delineation method performance when applied onto Patlak versus SUV images. However, the accuracy and precision of several (semi-) automatic tumor delineation methods have been studied previously using simulations [8] and clinical test-retest data [10]. Both articles showed that performance of tumor delineation methods are affected by several factors, such as scanner type, radiotracer, image noise, and tumor characteristics. It is generally accepted that pathology is the gold standard. Therefore, studies are needed and are currently performed that compare the tumor volumes obtained using (semi-)automatic delineation methods with pathology [11].

Although the Patlak analysis was performed on OSEMreconstructed images in order to reduce the levels of noise, Patlak images still showed a small fraction $(<1 \%)$ of voxels that had a negative slope, exclusively seen in non-tumor tissue locations. Correlation-coefficient 


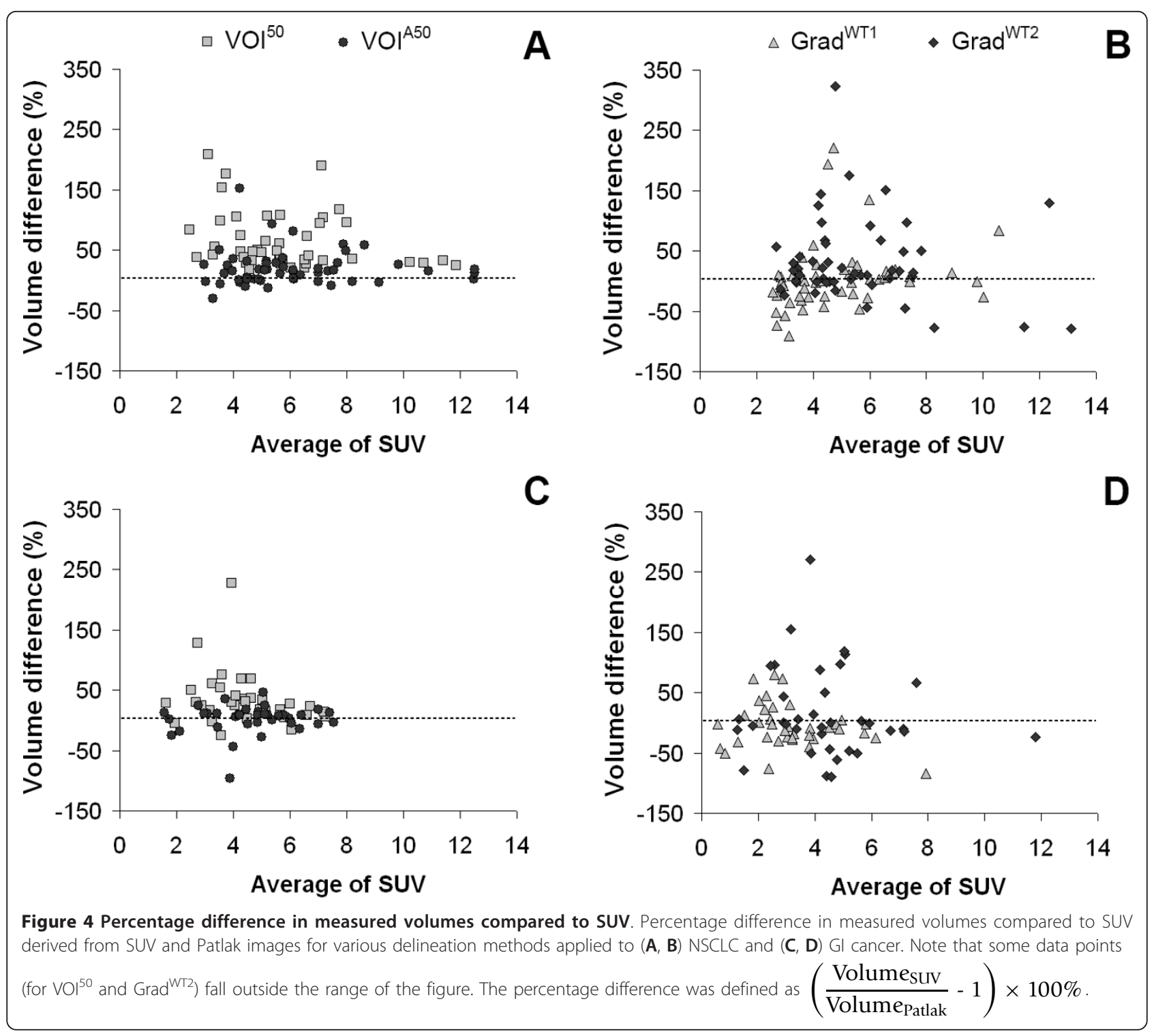

filtered parametric imaging, as proposed by Zasadny and Wahl [23], could potentially enhance the quality of the Patlak images and could be further investigated to improve the accuracy of automated tumor delineation. Despite the lack of using such a denoising method, our results were in line with those published by Visser et al. [6], and we could identify that tumor delineation methods that correct for local signal-to-background contrast or use gradients showed a better agreement in tumor volume assessment between Patlak and SUV images than those tumor delineation methods that did not.

\section{Conclusion}

Large differences may exist in metabolic volumes derived from static (SUV) and dynamic (Patlak) FDG image data. These differences depend strongly on the delineation method used. (Semi-)automatic tumor delineation methods that correct for local signal-to-background contrast or use gradients provide the most consistent results between SUV and Patlak images.

\section{Acknowledgements}

This study was performed within the framework of the Center for Translational Molecular Medicine (CTMM), AIRFORCE project (grant 030-103). Data used in this paper were derived in part from a study financed by Bristol-Myers Squibb. Patsuree Cheebsumon was supported by a scholarship from the National Science and Technology Development Agency of the Royal Thai Government.

\section{Author details}

'Department of Nuclear Medicine \& PET Research, VU University Medical Center, P.O. Box 7057, Amsterdam, 1007 MB, The Netherlands ${ }^{2}$ Department of Nuclear Medicine, Jeroen Bosch Hospital, 's-Hertogenbosch, 5223 GZ, The Netherlands ${ }^{3}$ Bristol-Myers Squibb, Princeton, NJ, 08543, USA 


\section{Authors' contributions}

PC performed the data analysis and data interpretation and was the main author of the manuscript. FHPVV performed the data interpretation, implemented some of the tumor delineation methods, and assisted in drafting the manuscript. MY implemented some of the tools to perform tumor delineations and critically reviewed the manuscript. $\mathrm{CJH}$ performed part of the data acquisition and critically reviewed the manuscript. LMV provided the PET image data and reviewed the manuscript. WH provided/ collected the PET image data and reviewed the manuscript. OSH reviewed the manuscript and approved its final content. AAL reviewed the manuscript, contributed to the intellectual content (supervision), and approved the final content of the manuscript. RB performed the study design, implemented some of the tumor delineation methods, supervised the project, and reviewed and approved the final content of the manuscript. All authors reviewed the collected data and interpretation, provided feedback for further research during the study, and approved the final submitted version of this manuscript

\section{Competing interests}

The authors declare that they have no competing interests.

Received: 7 November 2011 Accepted: 14 December 2011

Published: 14 December 2011

\section{References}

1. de Geus-Oei LF, van der Heijden HF, Corstens FH, Oyen WJ: Predictive and prognostic value of FDG-PET in nonsmall-cell lung cancer: a systematic review. Cancer 2007, 110:1654-1664.

2. Geets X, Lee JA, Bol A, Lonneux M, Gregoire V: A gradient-based method for segmenting FDG-PET images: methodology and validation. Eur J NuCl Med Mol Imaging 2007, 34:1427-1438.

3. Schaefer A, Kremp S, Hellwig D, Rube C, Kirsch CM, Nestle U: A contrastoriented algorithm for FDG-PET-based delineation of tumour volumes for the radiotherapy of lung cancer: derivation from phantom measurements and validation in patient data. Eur I Nucl Med Mol Imaging 2008, 35:1989-1999.

4. van Dalen JA, Hoffmann AL, Dicken V, Vogel WV, Wiering B, Ruers TJ, Karssemeijer N, Oyen WJ: A novel iterative method for lesion delineation and volumetric quantification with FDG PET. Nucl Med Commun 2007, 28:485-493.

5. Nestle U, Weber W, Hentschel M, Grosu AL: Biological imaging in radiation therapy: role of positron emission tomography. Phys Med Biol 2009, 54: R1-25.

6. Visser EP, Philippens ME, Kienhorst L, Kaanders JH, Corstens FH, de GeusOei LF, Oyen WJ: Comparison of tumor volumes derived from glucose metabolic rate maps and SUV maps in dynamic 18F-FDG PET. J Nucl Med 2008, 49:892-898

7. Hatt M, Cheze le RC, Albarghach N, Pradier O, Visvikis D: PET functional volume delineation: a robustness and repeatability study. Eur J Nucl Med Mol Imaging 2011, 38:663-672.

8. Cheebsumon $\mathrm{P}$, Yaqub M, van Velden FH, Hoekstra OS, Lammertsma AA Boellaard R: Impact of [(18)F]FDG PET imaging parameters on automatic tumour delineation: need for improved tumour delineation methodology. Eur I Nucl Med Mol Imaging 2011, 38:2136-2144.

9. Hatt M, Cheze-Le RC, Aboagye EO, Kenny LM, Rosso L, Turkheimer FE, Albarghach NM, Metges JP, Pradier O, Visvikis D: Reproducibility of 18FFDG and 3'-deoxy-3'-18F-fluorothymidine PET tumor volume measurements. J Nucl Med 2010, 51:1368-1376.

10. Cheebsumon $P$, van Velden FH, Yaqub M, Frings V, de Langen AJ Hoekstra OS, Lammertsma AA, Boellaard R: Effects of image characteristics on performance of tumor delineation methods: a test-retest assessment. J Nucl Med 2011, 52:1550-1558

11. Cheebsumon P, van Velden FHP, de Ruysscher D, van Elmpt W, Yaqub M, Hoekstra OS, Lammertsma AA, Boellaard R: Assessment of tumour volume in lung cancer: PET versus CT based methods [abstract]. Eur J Nucl Med Mol Imaging 2010, 37:s257.

12. Hoekstra CJ, Hoekstra OS, Stroobants SG, Vansteenkiste J, Nuyts J, Smit EF, Boers M, Twisk JW, Lammertsma AA: Methods to monitor response to chemotherapy in non-small cell lung cancer with 18F-FDG PET. J NuC Med 2002, 43:1304-1309.
13. Velasquez LM, Boellaard R, Kollia G, Hayes W, Hoekstra OS, Lammertsma AA, Galbraith SM: Repeatability of 18F-FDG PET in a multicenter phase I study of patients with advanced gastrointestinal malignancies. J Nucl Med 2009, 50:1646-1654.

14. Boellaard R, O'Doherty MJ, Weber WA, Mottaghy FM, Lonsdale MN, Stroobants SG, Oyen WJ, Kotzerke J, Hoekstra OS, Pruim J, et al: FDG PET and PET/CT: EANM procedure guidelines for tumour PET imaging: version 1.0. Eur J Nucl Med Mol Imaging 2010, 37:181-200.

15. Brix G, Zaers J, Adam LE, Bellemann ME, Ostertag H, Trojan H, Haberkorn U, Doll J, Oberdorfer F, Lorenz WJ: Performance evaluation of a whole-body PET scanner using the NEMA protocol. National Electrical Manufacturers Association. J Nucl Med 1997, 38:1614-1623.

16. Patlak CS, Blasberg RG: Graphical evaluation of blood-to-brain transfer constants from multiple-time uptake data. Generalizations. J Cereb Blood Flow Metab 1985, 5:584-590.

17. Cheebsumon P, Velasquez LM, Hoekstra CJ, Hayes W, Kloet RW, Hoetjes NJ, Smit EF, Hoekstra OS, Lammertsma AA, Boellaard R: Measuring response to therapy using FDG PET: semi-quantitative and full kinetic analysis. Eur $J$ Nucl Med Mol Imaging 2011, 38:832-842.

18. van der Weerdt AP, Klein $L$, Boellaard R, Visser CA, Visser FC, Lammertsma AA: Image-derived input functions for determination of MRGlu in cardiac (18)F-FDG PET scans. J Nucl Med 2001, 42:1622-1629.

19. Boellaard R, Krak NC, Hoekstra OS, Lammertsma AA: Effects of noise, image resolution, and ROI definition on the accuracy of standard uptake values: a simulation study. J Nucl Med 2004, 45:1519-1527.

20. Boellaard R: Standards for PET image acquisition and quantitative data analysis. J Nucl Med 2009, 50:11S-20S.

21. Frings V, de Langen AJ, Smit EF, van Velden FH, Hoekstra OS, van TH, Boellaard R: Repeatability of metabolically active volume measurements with 18F-FDG and 18F-FLT PET in non-small cell lung cancer. J Nucl Med 2010, 51:1870-1877.

22. Wanet M, Lee JA, Weynand B, De BM, Poncelet A, Lacroix V, Coche E, Gregoire V, Geets X: Gradient-based delineation of the primary GTV on FDG-PET in non-small cell lung cancer: a comparison with thresholdbased approaches, CT and surgical specimens. Radiother Oncol 2011 98:117-125.

23. Zasadny KR, Wahl RL: Enhanced FDG-PET tumor imaging with correlationcoefficient filtered influx-constant images. J Nucl Med 1996, 37:371-374.

doi:10.1186/2191-219X-1-35

Cite this article as: Cheebsumon et al: Measurement of metabolic tumor volume: static versus dynamic FDG scans. EJNMMI Research 2011 $1: 35$

\section{Submit your manuscript to a SpringerOpen ${ }^{\circ}$ journal and benefit from:}

- Convenient online submission

- Rigorous peer review

- Immediate publication on acceptance

- Open access: articles freely available online

- High visibility within the field

- Retaining the copyright to your article

Submit your next manuscript at $>$ springeropen.com 